\title{
Reconfigurable optical multiplexer based on liquid crystals for polymer optical fiber networks
}

\author{
P.C. LALLANA*, C. VÁZQUEZ, J.M.S. PENA, and R. VERGAZ
}

Electronics Technology Department, Carlos III University of Madrid, C/Butarque 15, 28911 Leganés, Madrid, Spain

In this work, different novel $3 \times 1$ multiplexer structures for being used in polymer optical fiber networks are proposed. Designs are compact, scalable, and of low consumption, capable of operating in a large wavelength range simultaneously 660 , 850 , and $1300 \mathrm{~nm}$, due to the use of nematic liquid crystal cells. Light that comes from each input port is handled independently and eight operation modes are possible.

Control electronics has been made using a programmable integrated circuit. Electronic system makes available the managing of the optical stage using a computer. An additional four optical sensors have been included for allowing the optical status checking.

Finally, a polarization independent multiplexer has been implemented and tested. Insertion losses less than $4 \mathrm{~dB}$ and isolation better than $23 \mathrm{~dB}$ have been measured. In addition, 30-ms and 15-ms setup and rise times have been obtained. The proposed multiplexer can be used in any polymer optical fiber network, even in perfluorinated graded index one, and it can be specially useful in optical sensor networks, or in coarse wavelength division multiplexing networks.

Keywords: multiplexer, liquid crystals, polymer optical fiber, coarse wavelength division multiplexing.

\section{Introduction}

Nowadays, the number of applications that use polymer optical fibers (POF) is increasing quickly. In short distances, less than one hundred meters, they have large bandwidth, two visible wavelength division multiplexings have been implemented over 20 meters POF in Ref. 1, allowing full duplex communication. Another visible/near infrared wavelength division multiplexing over POF application is shown in Ref. 2, a duplex data and a voice signal transmission are done at the same time. In addition, these fibers are lightweight, immune against electromagnetic interferences (EMI) and of easy installation. Therefore, POF is being used in video transmission in medical equipment, or in multimedia applications for civil aviation and high range cars [3], or in optical sensor networks [4,5], where due to its safety nature, they are made of inert materials and can be used in flammable atmospheres where no risk of explosion is caused even under malfunction operation.

Perfluorinated graded index POF (PF GI-POF) has a low loss wavelength range from 500 to $1300 \mathrm{~nm}[6,7]$, allowing the implementation of coarse wavelength division multiplexing (CWDM) in high-speed networks and in reconfigurable optical networks for critical applications. Due to the amount of optical networks, and their development during last years, it could be interesting to perform optical switching, even it must be necessary in redundant networks used in critical applications where safety is fundamental, and redundant paths are needed. Many different technologies have been reported for optical switching.

Nowadays, micro-electromechanical systems (MEMs) $[8,9]$ that use mobile parts are attractive because of their large integration scale, their fiber-to-fiber coupling, high crosstalk and speed. An additional large displacement optical fiber $1 \times 2$ switch is presented in Ref. 10. Switches based on liquid crystal cells [11-16] are used frequently instead of other technologies, they do not have mobile parts, need low excitation voltage, and have low power consumption. However, most of these systems have a complex structure involving a great amount of components [11-13]. In Ref. 14 , less components are used but a fiber optic circulator is needed which complicates its integration. A liquid crystal based optical switch using also polarizing beam splitter is given in Ref. 15, but it only works at a single wavelength. Another switch structure using a liquid crystal cell and a polarizing beam splitter capable of working in a wide wavelength range can be seen in Ref. 16.

Liquid crystal based switches work with nematic LC (NLC) $[11,13,15,17]$ or ferroelectric LC (FLC) [12,18]. Last ones exhibit fast response times, but they can operate in a smaller wavelength range because FLC cell thickness $d$ should be different in order to obtain a $90^{\circ}$ polarization switch at each wavelength. The birefringence of this material $\Delta n$, depends on the wavelength $\lambda$, and the $\Delta n \cdot \lambda$ product 
must be a constant to get the polarization switch desired. Instead of that, only Mauguin's regime $(\Delta n \cdot d / \lambda>>1)$ must be kept if nematic liquid crystal cells are used. Proposed structures in this work can operate with both types of liquid crystals, but nematic liquid crystals have been selected for the implementation because Mauguin's regime could be fulfilled simultaneously at the desired wavelengths 660,850 , and $1300 \mathrm{~nm}$.

With these issues in mind, in sec. 2, different compact, reconfigurable and broadband $1 \times 3$ multiplexer schemes are proposed for being used in POF (even PF GI-POF) networks. All designs are formed by a reduced number of components, where nematic liquid crystal cells and polarizing beam splitter are included. A deep description of the optical and electronic stage implemented in the $1 \times 3$ polarization independent multiplexer is given in sec. 3 , including a brief description of the multiplexer's control software programme. Section 4 includes measurements done for testing the implemented design. Finally, some conclusions are given.

\section{Optical multiplexer structure}

An optical $N \times 1$ multiplexer is a device capable of joining $N$ input optical fibers in a single output fiber. Common optical multiplexers combine different wavelength sources in the same optical fiber, thus, wavelength division multiplexing (WDM) over a fiber is performed. In this kind of multiplexers, input ports are not interchangeable, each input port must be excited by a pre-allocated wavelength source. In this work, we propose $3 \times 1$ multiplexers where inputs are wavelength independent, as they work in the same way for different wavelengths. Time division multiplexing (TDM) can also be implemented by means of these devices, even a mixture of a TDM and a WDM, i.e., different fibers involving WDM can be temporally multiplexed into a single fiber. Figure 1 shows a generic symbol of a $3 \times 1$ multiplexer, it is composed by 3 inputs and only an output port.

Different optical $3 \times 1$ multiplexer architectures are described. Proposed schemes can manage light that comes from each input independently, thus eight different setups are possible:

- only light from one port reach the output (three setups, one for each port),

- light from two of the three input ports gets to the output (three setups, one for each pair of ports),

- light of all the input fibers passes through the device (one status),

- there is no light in the output fiber (last status).

This configuration makes possible more flexibility in applications than wavelength multiplexer, because it can also be used as a time division multiplexer independently of the input light wavelength.

Active elements in the designs are twisted nematic liquid crystal cells (TN-LC) working at 660, 850, and 1300 $\mathrm{nm}$. These LC cells modify their optical behaviour depend-

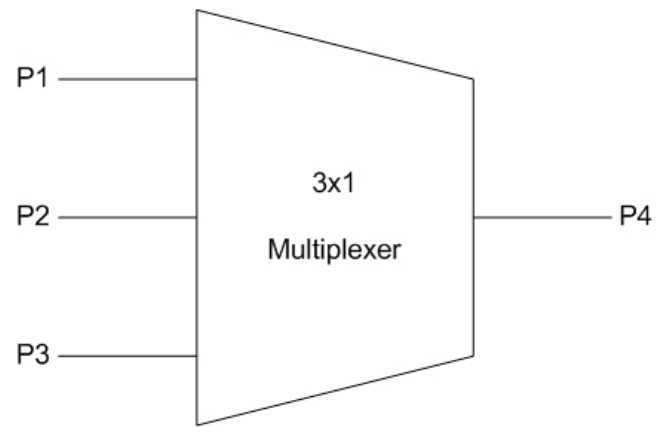

Fig. 1. Symbol of a $3 \times 1$ multiplexer.

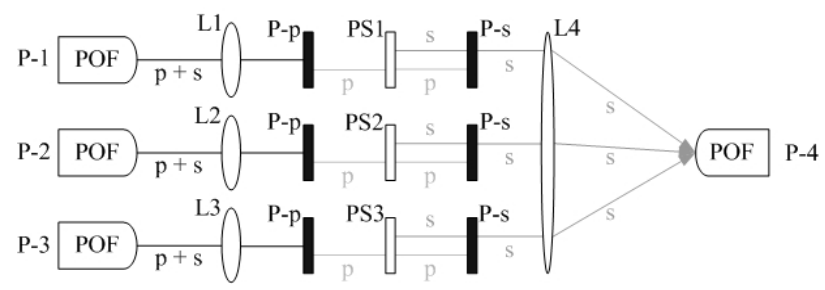

Fig. 2. Basic scheme of a $3 \times 1$ multiplexer.

ing on the voltage applied to them. When they are not excited, the input light polarization is shifted, but, when an electric field is applied to them, the input light polarization is maintained. Thus, light passing can be controlled with the use of liquid crystal cells in combination with the convenient polarizers.

Proposed multiplexers are designed for being used with polymer optical fiber (POF). Light coming out from POF has a random polarization. The schemes shown in Figs. 2 and 3 are able to manage only one light polarization, then, having insertion losses greater than $3 \mathrm{~dB}$. The other scheme,

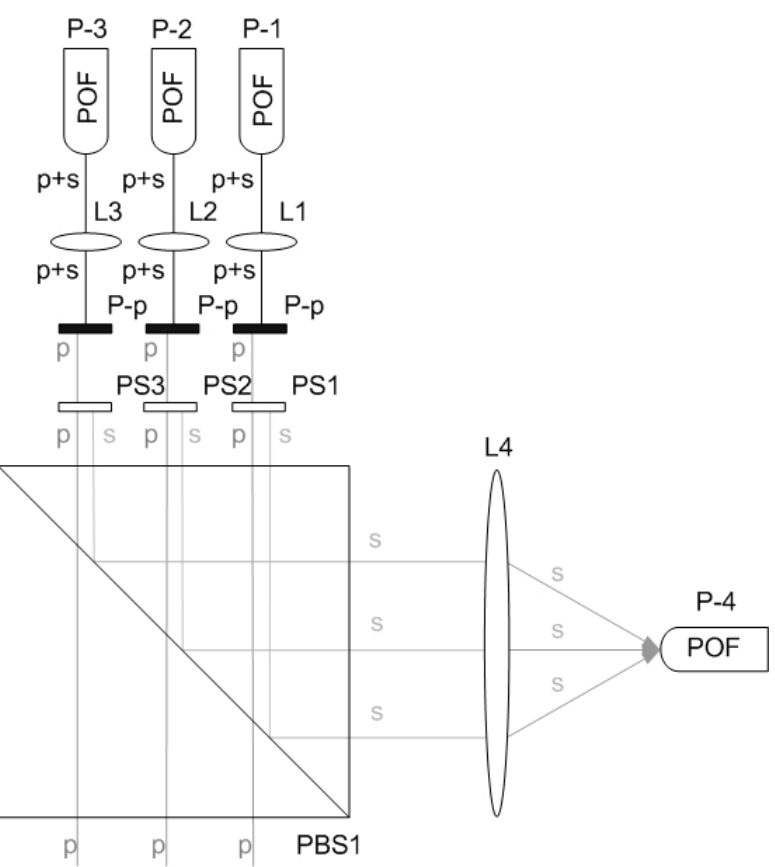

Fig. 3. Alternative scheme of a $3 \times 1$ multiplexer. 
shown in Fig. 4, can handle the two light polarizations, having less insertion losses, and being polarization independent.

The simplest proposed structure, shown in Fig. 2, has a reduced number of elements and the input polarizers $\left(\mathrm{P}_{-\mathrm{p}}\right)$ filter out half of the input light. Three liquid crystal cells (PS1, PS2, and PS3) can rotate or not each input port light polarization, and depending on LC status, light would pass or not through the second polarizer $\left(\mathrm{P}_{-\mathrm{s}}\right)$. Output lens (L4) is used for focusing all the beams in the output fiber (P-4). Input lenses (L1, L2, and L3) collimate polymer optical fiber output light.

According to Fig. 2, each input light reaches the output fiber when the corresponding liquid crystal cell is in OFF status, so no electrical signal is applied to LC electrodes. After the first polarizer, the remaining polarization is shifted and passes through the second polarizer and gets to the multiplexer output. Otherwise, when the liquid crystal cell is in ON status, input light polarization remains and it is filtered in the second polarizer, in this way, input light does not reach the output. Each liquid crystal cell is controlled independently, and in this way the eight multiplexer operation modes can be configured.

An alternative scheme, working in the same manner, is shown in Fig. 3. In this proposal, a polarizing beam splitter acts like the second set of polarizers of Fig. 2. The isolation, light that passes through the multiplexer when it is inactive, is improved because polarizing beam splitter filters more efficiently the polarized light.

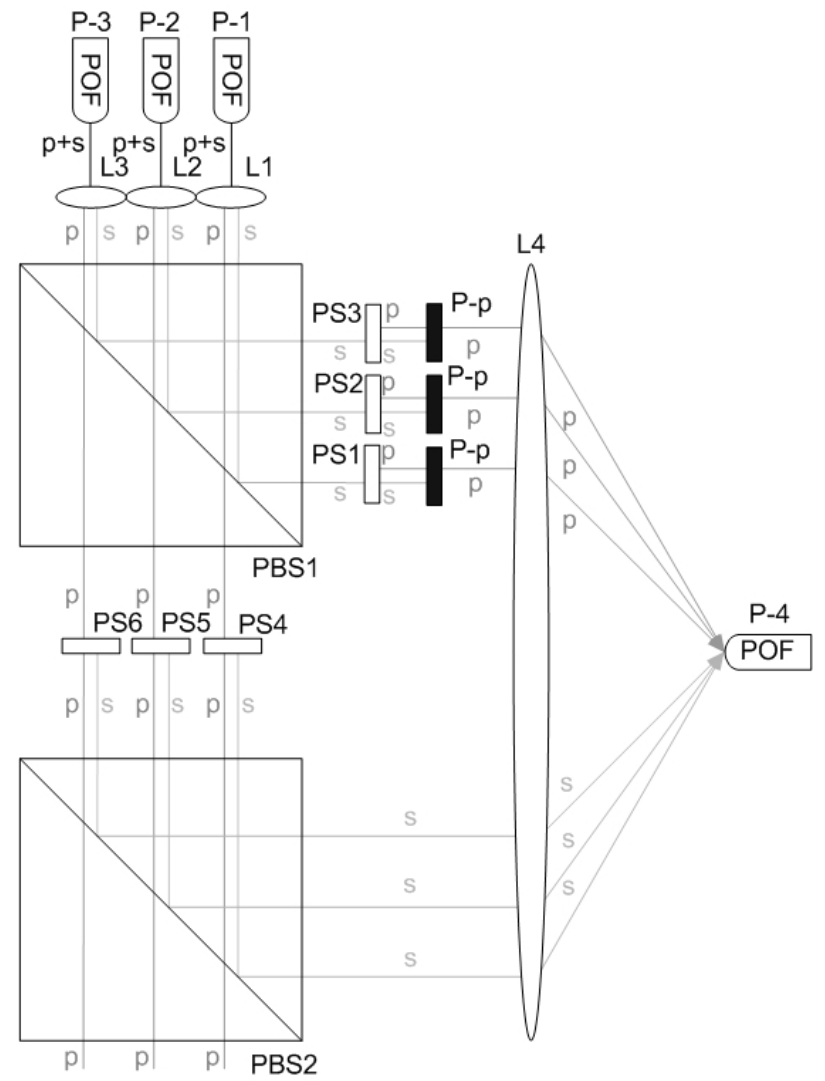

Fig. 4. Polarization independent scheme of a $3 \times 1$ multiplexer.
The last structure, shown in Fig. 4, is explained in the next section. It can manage the two polarizations and at least an insertion losses improvement of $3 \mathrm{~dB}$ is expected. This design has been implemented, and some measurements are shown in sec. 4.

\section{Architecture of the implemented multiplexer}

In previous section, two polarization dependent multiplexer schemes have been described, a $50 \%$ of each input light is filtered. Reduction of insertion losses is possible if the multiplexer can act over the two polarizations. In this section, a more complex, but polarization independent, design is explained. The input beam is divided into two polarizations and each polarization is treated by different liquid crystal cells but in the same way.

The proposed multiplexer can be divided in optical and electrical hardware sections, Fig. 5 shows its block diagram. Optical part manages the light that comes from each input port, and it is in charge of allowing its pass through the device to the output port, or not. Electronic part controls the status of the optical part and allows the user to control the multiplexer by means of a computer using specific software. Each part is described in the next subsections.

\subsection{Optical scheme}

The proposed optical scheme is shown in Fig. 4. It has three input ports $\mathrm{P}-1, \mathrm{P}-2$, and $\mathrm{P}-3$, and a single output port $\mathrm{P}-4$. The device elements are two polarizing beam splitters (PBS 1 and PBS 2), six liquid crystal cells (PS1-PS6), three polarizers $\left(\mathrm{P}_{\mathrm{p}}\right)$, and lenses $(\mathrm{L} 1-\mathrm{L} 4)$.

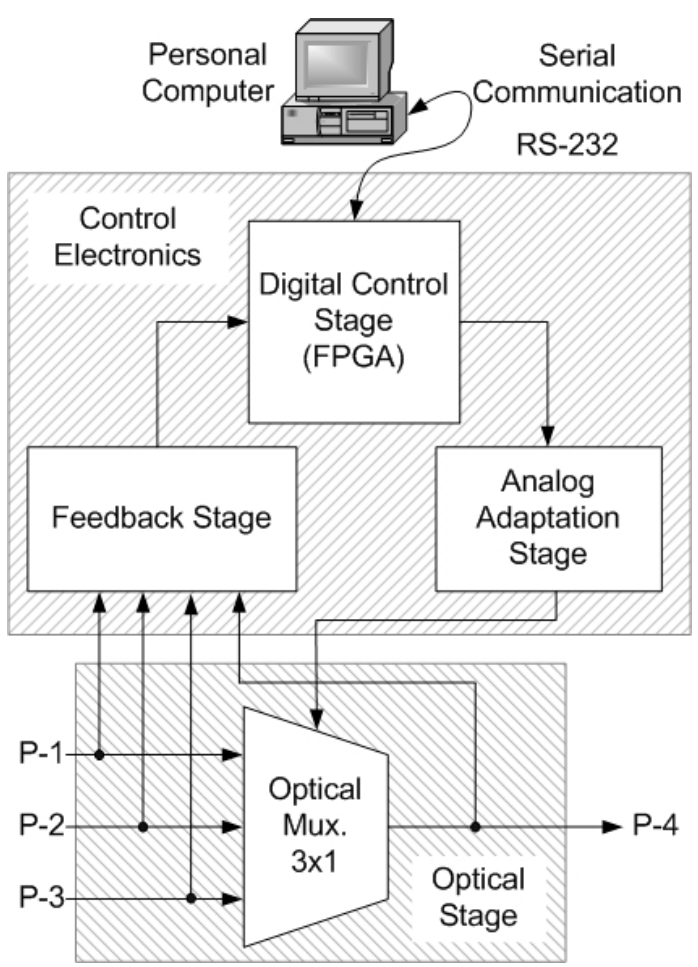

Fig. 5. Block diagram of a $3 \times 1$ multiplexer proposed. 
Polarizing beam splitters divide the non-polarized incident light beam in two different polarized beams, s-polarization is deflected $90^{\circ}$ while p-polarization passes through the device. Polarizers eliminate s-polarization of its incident light. Three lenses (L1-L3) are used for collimating the light that comes from each input port and the remaining lens (L4) is used for focalizing light in the output fiber.

Light coming from each input port is divided in two polarized beams when passes through the first PBS. Three of the liquid crystal cells (PS1, PS2, and PS3) act over p-polarization beams and the others operate over s-polarization beams (PS4, PS5, and PS6). In addition, liquid crystal cells work in pairs, one for each input port, PS1-PS4 act over light coming from port one, PS2-PS5 over port two, and PS3-PS6 over port three.

The second beam splitter acts as an analyzer, depending on the corresponding liquid crystal status, the beam is deflected $90^{\circ}$, s-polarization, or continues straight, p-polarization. On the other path, the polarizers do the same work, depending on the corresponding liquid crystal status, light passes through the polarizers, p-polarization, or not, s-polarization. Finally, the last lens focalises all the available light beams in the output port.

According to the scheme, when the corresponding pair of liquid crystals is in OFF state, no voltage is applied to them, polarization is switched and each optical path continues to the output fiber. One beam is deflected $90^{\circ}$ and the other passes through the polarizer. When the pair of liquid crystals is in ON state, incident light polarization is maintained and the light does not reach the output fiber. One beam passes through the second polarizing beam splitter and it gets lost, the other beam is filtered in the polarizer.

An optical path is active when light that comes from the corresponding port reaches the output port, and it is inactivate otherwise. In this way, a port is active when the appropriate pair of liquid crystals is in OFF state, and it is inactive when those liquid crystals are in ON state.

\subsection{Control electronics}

Control of the $3 \times 1$ multiplexer is carried out by electronics. Six output signals are generated for exciting liquid crystal cells. A serial communication is provided for modifying the status of the multiplexer using a computer. Additional feedback information is possible thanks to four optical sensors. In this way, if a 10/90 optical coupler is used in each port of the multiplexer, the optical power can be measured, and proper operation of the multiplexer is checked.

Figure 5 also shows the block diagram of the electronic part. The design is divided in three different stages, digital, analogue, and feedback stages. Digital stage is the centre of the design and generates the signals needed for controlling the rest of the system. Analogue adapt stage modifies signals voltage for liquid crystal requirements. Feedback stage converts optical power coming from each multiplexer port to digital values, serving as entries for the digital part.

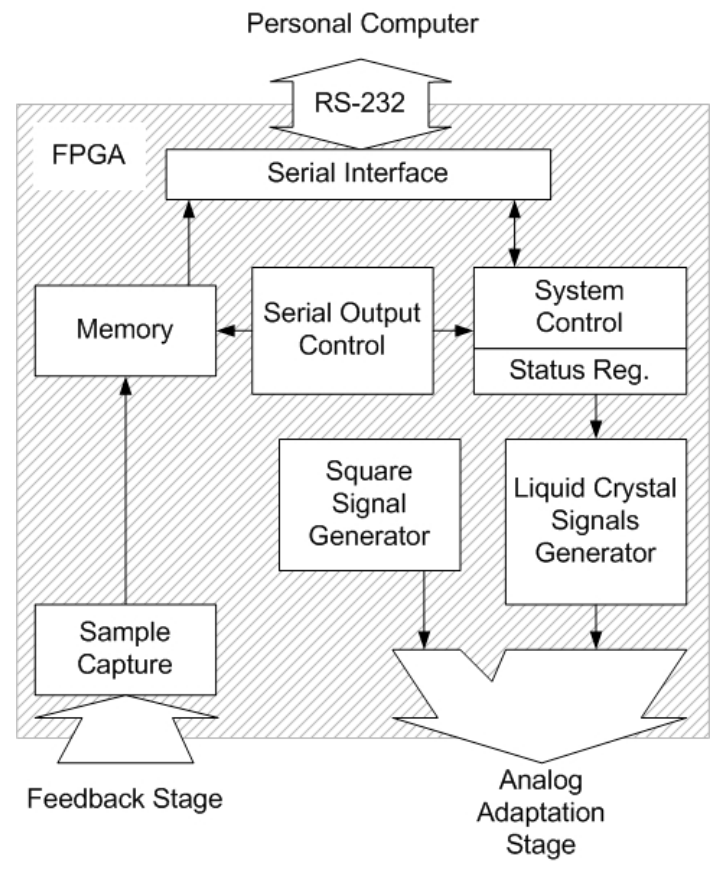

Fig. 6. Block diagram of the digital stage.

Digital part is implemented in a programmable integrated circuit, a field programmable gate array (FPGA). In these devices, a hardware description of the electronics is programmed, and multiple configurations are allowed, for this reason, the improving and debugging of the designs is quite easy.

Scheme of the digital stage is shown in Fig. 6. The status register stores the multiplexer operation mode, one of the eight possible setups. An $8-\mathrm{kHz}$ square wave is generated by the square signal generator and six different signals are used to control their state. The square wave will be applied to the liquid crystal pixels that are active, in ON status for an inactive port, and no excitation, zero volts, when the cells are in OFF status, for an active port.

Analogue adapt stage is in charge of applying $8 \mathrm{-kHz}$ square wave to the corresponding liquid crystal cells depending on the output provided by the digital part. A voltage conversion is carried out, in this way the continuous component of the square wave is eliminated, because it could damage the LC cell, and its amplitude is adjusted to the adequate value for exciting liquid crystal cells. Threevolt amplitude is enough for the switching of the used liquid crystal cells, but the implemented design allows modifying this value.

In the feedback stage, a four-channel, parallel, analogue to digital converter transforms the optical sensor measurements in a digital eight bits bus. Digital stage continuously request samples to the converter and stores them in an inner memory.

Control of the multiplexer is done by means of a computer using RS-232 serial communication. A serial interface and an additional serial output control are available for allowing data transfer. A subsequent electronics adapt volt- 
age values to the protocol specifications. By this mean, multiplexer operation mode can be switched and the user can ask for the optical power measurements and check the proper operation of the device.

\subsection{Software description}

A serial communication allows remote operation of the device. In this way, an application has been programmed for allowing the control of the multiplexer using a computer. Users can select which input ports are active or inactive. Due to the device's implementation, there are no restrictions about how many optical paths are active at the same time, even the three ports can be active at the same time.

The program is sending and requesting information continuously to the device. In this way, every modification of the configuration can be sent and checked immediately. In the same way, the user can select which optical measurements are displayed in the program window.

\section{Measurements}

Static and dynamic measurements have been carried out for characterizing switching voltage, insertion losses and isolation of the multiplexer, at a single wavelength. A function generator and a laser have been used for all these measurements. Experimental set up is designed to generate signals as the ones to be provided by the designed electronics.

\subsection{Set up}

Figure 7 shows the experimental set up used for doing the measurements. Each port has been measured independently for avoiding interchannel noise. A visible diode laser at $650 \mathrm{~nm}(620-680 \mathrm{~nm})$ and $5.0 \mathrm{~mW}(7 \mathrm{dBm})$ from Power Technology Inc. has been used. An iris diaphragm has been used at the laser's output for limiting the beam's spot, and in this way no light is out of the active part in each liquid crystal cell. There are no input lenses (L1, L2, and L3) because the diode laser includes collimation optics. A 97-cm length plastic optical fiber joins multiplexer's output with the measuring device to be characterized. Output beam is

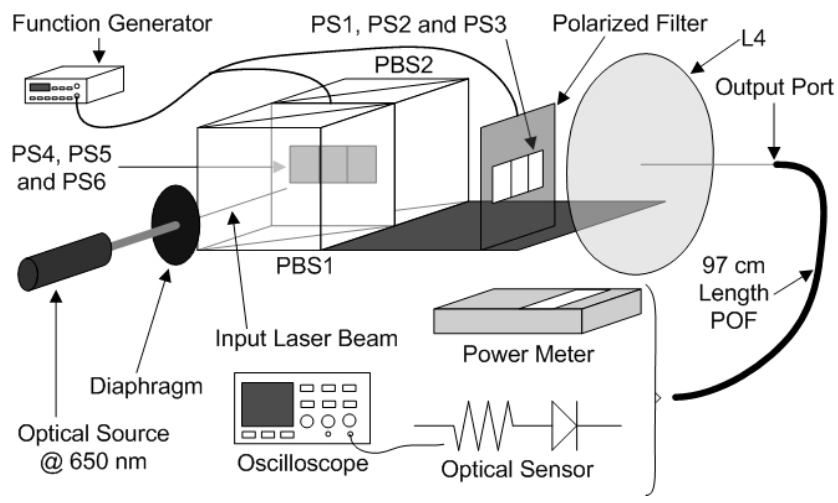

Fig. 7. Set up used for measurements. focused in the optical fiber by means of L4 lens. Two different measurement systems have been utilized, on one hand, a 557B Rifocs commercial optical power meter, on the other hand, an Infineon SFH $350 \mathrm{~V}$ phototransistor with a $100-\Omega$ resistance. A Hewlett Packard function generator has been used for exciting liquid crystal cells. And a Tektronix digital oscilloscope is utilized for measuring voltage when the phototransistor acts as optical sensor.

\subsection{Static measurements}

This section refers to the measurements done when the excitation of the liquid crystal cells is maintained during a long period of time. The same $8-\mathrm{kHz}$ square wave is applied to the pair of liquid crystal cells that form an optical path. Signal's amplitude is modified in each measurement from $0 \mathrm{~V}$ to $4 \mathrm{~V}$. In this way, switching voltage of liquid crystal cells, insertion losses and isolation can be obtained.

Figure 8 shows the transmission obtained for the three ports. It can be observed that the three curves are similar. Ports are active from $0 \mathrm{~V}$ to $1 \mathrm{~V}$ and they can be considered that ports are inactive when the amplitude exceeds $3 \mathrm{~V}$. In this way, insertion losses of the multiplexer are less than 4 $\mathrm{dB}$, and the isolation, relationship between active status and inactive status of each port, is more than $23 \mathrm{~dB}$. The measured optical power after the diaphragm was $-6.9 \mathrm{dBm}$. Table 1 shows the losses and isolation measurements obtained for each port.

It is supposed that at zero volts a maximum of transmission is expected, and when the liquid crystal cell is switched on, the transmission would be minimum, but it does not happen exactly in this way, it seems that the maximum and the minimum are a bit shifted. This may occur because there is not a good alignment. When the voltage begins to increase, liquid crystal molecules start to rotate incident light polarization, and there is more transmission, in the same way, after liquid crystal cell are completely switched, there is a minimum of transmission. For this reason it can

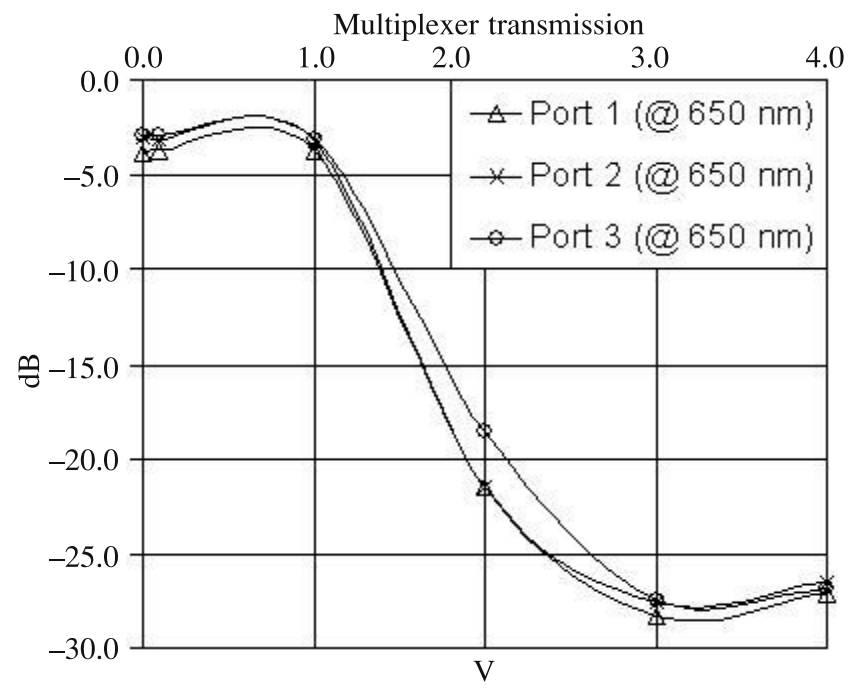

Fig. 8. Multiplexer losses for static measurements. 
be supposed that there is a small error in the alignment between the two polarizing beam splitters, the polarizer and the liquid crystal cells. Correcting this problem, at least a decibel improvement can be achieved in both, insertion losses and isolation.

Table 1. Insertion losses and isolation of a $1 \times 3$ multiplexer.

\begin{tabular}{ccc}
\hline Port & Insertion $(\mathrm{dB})$ & Isolation $(\mathrm{dB})$ \\
\hline P1 & 3.9 & 23.2 \\
P2 & 3.0 & 23.5 \\
P3 & 2.9 & 23.9 \\
\hline
\end{tabular}

\subsection{Dynamic measurements}

This section refers to the measurements when optical paths are switching on/off repeatedly. In this case, an $8-\mathrm{kHz}$ and $3-\mathrm{V}$ amplitude square wave is applied during $50 \mathrm{~ms}$ and 0 $\mathrm{V}$ for other $50 \mathrm{~ms}$, in other words, a square envelope of 10 $\mathrm{Hz}$ is used for modulating the $8-\mathrm{kHz}$ square wave. As in previous section, the same signal is applied to the pair of liquid crystal cells that form each optical path. A digital oscilloscope and an optical sensor (Infineon SFH $350 \mathrm{~V}$ phototransistor with a $100 \Omega$ resistance) is used to measure optical power. Figure 9 shows an image captured from the digital oscilloscope, liquid crystal's excitation signal is displayed in the upper part of the picture, and the response of the multiplexer is viewed in the lower section.

The same excitation has been applied to the three multiplexer ports and similar results are obtained. Figure 10 shows the optical power losses measured, the upper one corresponds to port two, while the other ports have equivalent performance. In Table 2, several temporal parameters are shown, rise time is less than $20.5 \mathrm{~ms}$ and down time is less than $5.2 \mathrm{~ms}$. $t_{o n}$, time interval since voltage is switching off until optical path is active, is less than $30.5 \mathrm{~ms}$ and $t_{\text {off }}$, time interval since voltage is applied until optical path is inactive, is better than $12 \mathrm{~ms}$. These measurements are in accordance with liquid crystal properties, it is common that relaxation times are larger than molecular orientation times, and the values obtained correspond with previous characterizations. On the other hand, it seems that insertion losses are slightly greater than in static measurements, but they are always less than $4.5 \mathrm{~dB}$.

Table 2. Response time of a $1 \times 3$ multiplexer.

\begin{tabular}{ccccc}
\hline Port & $t_{\text {on }}(\mathrm{ms})$ & $t_{\text {rise }}(\mathrm{ms})$ & $t_{\text {off }}(\mathrm{ms})$ & $t_{\text {down }}(\mathrm{ms})$ \\
\hline P1 & 29.2 & 20.0 & 12.0 & 5.2 \\
P2 & 30.4 & 20.4 & 12.0 & 4.8 \\
P3 & 28.8 & 14.8 & 10.8 & 4.8 \\
\hline
\end{tabular}

The proposed multiplexer can work at different wavelengths, 660,850 , and $1300 \mathrm{~nm}$. It has been only tested at $660 \mathrm{~nm}$, but a characterization of liquid crystal cells, with similar properties to the ones used in the multiplexer, has

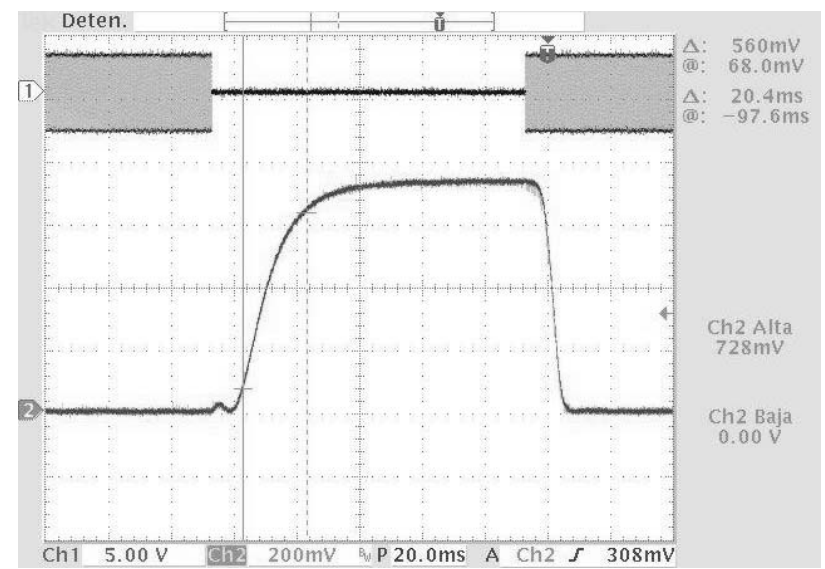

Fig. 9. Excitation signal and multiplexer response.

been previously made at $808 \mathrm{~nm}$ [16]. The measurements have been made with the liquid crystal cell placed between two polarizing beam splitters acting as cross-polarizers. Figure 11 shows the transmission obtained when different amplitudes and frequency square waves are applied to the liquid crystal cells. Insertion losses of $0.9 \mathrm{~dB}$ and an isolation of $12 \mathrm{~dB}$ have been measured at $808 \mathrm{~nm}$, while $0.7 \mathrm{~dB}$ and $14 \mathrm{~dB}$ were obtained at $660 \mathrm{~nm}$, respectively. Relaxation time of $33 \mathrm{~ms}$ was obtained for the tested cells. Values obtained at $660 \mathrm{~nm}$ are similar between samples used in the multiplexer and the ones tested previously, for this reason is expected that their behaviour at $808 \mathrm{~nm}$ would be also similar. A subsequent characterization at $1300 \mathrm{~nm}$ will be made in the near future.

\section{Conclusions}

Different configurations for a $3 \times 1$ multiplexer with eight operation modes have been proposed with simple, compact, and scalable structures. They have wide wavelength range operation thanks to the use of twisted nematic liquid crystal cells that are capable of working at 660, 850, and $1300 \mathrm{~nm}$, even at the same time. In addition, these devices

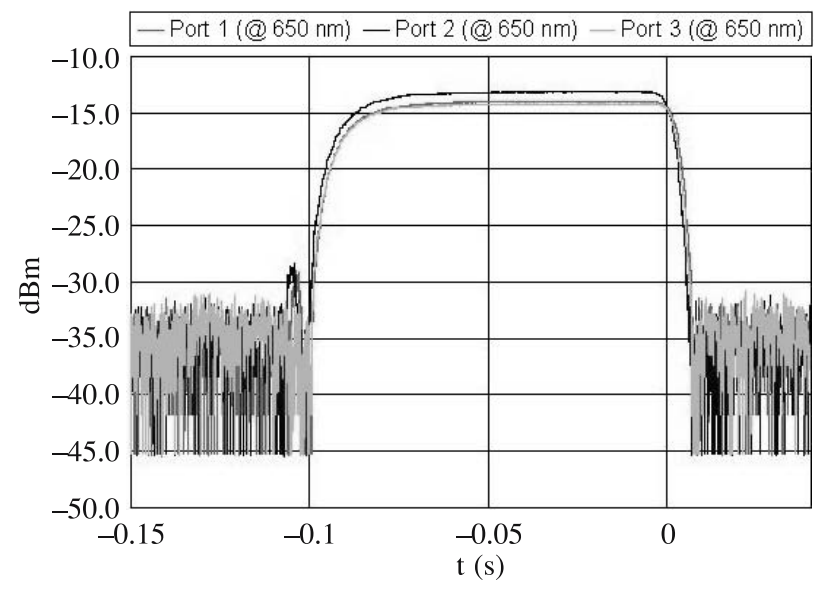

Fig. 10. Multiplexer losses from dynamic measurements. 


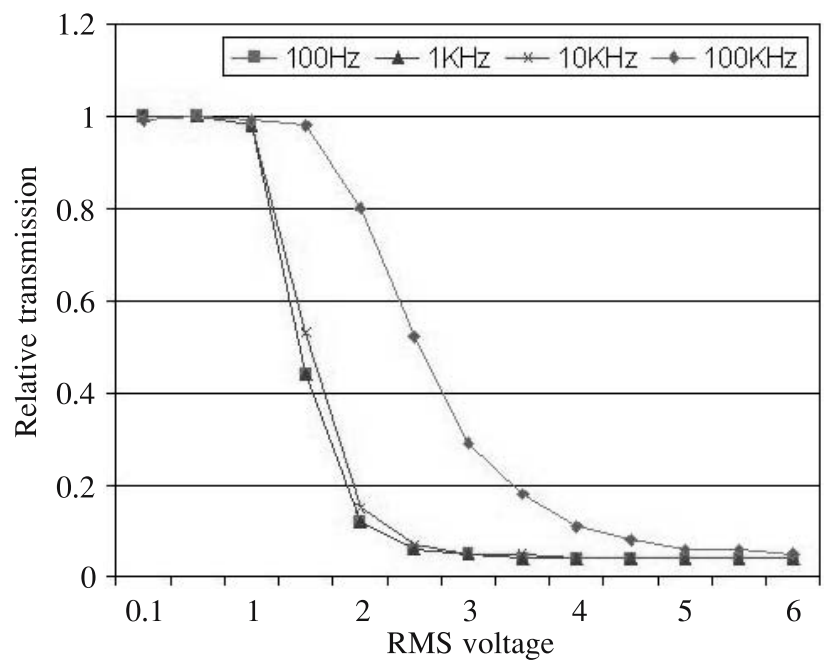

Fig. 11. Relative transmission of a liquid crystal cell at $850 \mathrm{~nm}$.

need low excitation voltages and have low power consumption.

A polarization independent configuration has been developed, and experimentally tested. Insertion losses less than $4 \mathrm{~dB}$ and isolation better than $23 \mathrm{~dB}$ have been measured, but this value could be improved with a better alignment. In addition, 30-ms and 15-ms setup and rise times have been obtained.

Control electronics using a reconfigurable device has been implemented, capable of modifying the multiplexer operation. In addition, the status of the multiplexer can be checked thanks to four optical sensors that allow the measurement of the optical power in each port. A computer application has been programmed allowing the remote control of the multiplexer.

$\mathrm{N}$-input multiplexer can be implemented using larger polarizing beam splitters, more liquid crystal cells and a bigger output lens. The developed electronics can be easily modified to allow for the new amount of inputs due to the use of a programmable integrated circuit, and duplicating or multiplexing analogue electronic circuits.

Finally, the proposed scheme can be used in optical networks as a switching device. It could be interesting its use in coarse wavelength division multiplexing (CWDM) networks due to wide bandwidth or even in optical sensor networks because it can be used as a typical electronic multiplexer but in optical domain.

It can be interesting the use of a Savart plate, which separates light's polarization in two parallel beams, instead of the polarizing beam splitters. In this way, less optical components are needed and more linear device behaviour is expected.

\section{Acknowledgements}

This work has been supported by the CICYT:TIC200303783 and TEC2006-13273-C03-03-MIC (Ministry of Science and Education), UC3M: FAVICOVIS (University
Carlos III of Madrid), CAM:FACTOTEM-CM (S-0505/ ESP/000417) (Comunidad de Madrid) and SAMPA (TNVPM-EU) Projects. We wish to thank to J.M. Otón and X. Quintana for the manufacturing of the liquid crystal cells used in the prototype, and to Juan José Cañadas Rufo for the development of the control electronics.

\section{References}

1. M. Yonemura, A. Kawasaki, S. Kato, M. Kagami, and Y. Inui, "Polymer waveguide module for visible wavelength division multiplexing plastic optical fiber communication", Optics Lett. 30, 2206-2208 (2005).

2. Y. Zhang, H. Ma, T. Zhang, Z. Wang, D. Wang, R. Zheng, H. Yang, and H. Ming, "Study of coarse wavelength division multiplexing using polymer optical fiber transmission window", Proc. SPIE 5644, 835-839 (2005).

3. T. Kibler, S. Poerl, G. Böck, H.P. Huber, and E. Zeeb, “Optical data buses for automotive applications", J. Ligthwave Technol. 22, 2184-2199 (2004).

4. S.F. Knowles, B.E. Jones, S. Purdy, and C.M. France, "Multiple microbending optical-fiber sensor for measurements of fuel quantity in aircraft fuel tanks", Sens. Actuators A68, 320-323 (1998).

5. C. Vázquez, A.B. Gonzalo, S. Vargas, and J. Montalvo, "Multi-sensor system using plastic optical fibers for intrinsically safe level measurement", Sens. Actuators A116, 22-32 (2004).

6. J. Goudeau, G. Widawski, and B. Bareel, "GI-POF for GB Ethernet links", $13^{\text {th }}$ Int. Plastic Optical Fibres Conf. 76, (2004).

7. Y. Koike and T. Ishigure, "High-speed, low-loss polymer optical fibers", EEP 25, Workshop on Mechanical Reliability of Polymeric Materials and Plastic Packages of IC Devices. ASME, 19-29 (1998).

8. C. Marxer and N.F. De Rooij, "Micro-op-mechanical $2 \times 2$ switch for single-mode fibers based on plasma-etched silicon mirror and electrostatic actuation", J. Lightwave Techn. 17, 2-6 (1999).

9. R.A. Jensen, "Comparing of optical switching technologies for intelligent optical networks", LEOS'02, Vol. I, 230-231 (2002).

10. M.I. Bhuiyan, Y. Haga, and M. Esashi, "Design and characteristics of large displacement optical fiber switch", $J$. Quantum Electronics 41, 242-249 (2005).

11. R.A. Soref, "Low-cross-talk $2 \times 2$ optical switch", Optics Lett. 6, 275-277 (1981).

12. N. Riza and S. Yuan, "Low optical interchannel crosstalk, fast switching speed, polarization independent $2 \times 2$ fibre optic switch using ferroelectric liquid crystals", Electr. Lett. 5, 1341-1342 (1998).

13. Y. Fujii, "Low-crosstalk $2 \times 2$ optical switch composed of twisted nematic liquid crystal cells", IEEE Photonics Technology Letters 5, 715-718 (1993).

14. S. Sumriddetchkajorn, N. Riza, and D. Sengupta, "Liquid crystal-based self-aligning $2 \times 2$ wavelength routing module", Opt. Engin. 40, 1521-1528 (2001).

15. N.A. Riza "High-optical isolation low-loss moderate-switching-speed nematic liquid-crystal optical switch", Optics Lett. 19, 1780-1782 (1994). 
16. C. Vázquez, J.M.S. Pena, and A.L. Aranda, "Broadband $1 \times 2$ polymer optical fiber switches using nematic liquid crystals", Optics Comm. 224, 57-62 (2003).

17. F. Pain, R. Coquillé, B. Vinouze, N. Wolffer, and P. Gravey, "Comparison of twisted and parallel nematic liquid crystal polarisation controllers. Applications to a $4 \times 4$ free space optical switch at $1.5 \mathrm{~mm}$ ", Optics Comm. 139, 199-204 (1997).

18. A. Riza and S. Yuan, "Reconfigurable wavelength add-drop filtering based on a banyan network topology and ferroelectric liquid crystal fiber-optic switches", J. Light. Tech. Lett. 17, 1575-1584 (1999). 